\title{
Facets on Fatigue Fractures
}

\author{
Nicholas E. Cherolis \\ Rolls-Royce Corporation, Materials Technology, Indianapolis, Indiana, USA
}

One of the most common failure modes for aerospace components is fatigue. In the classical literature the fatigue event is broken down into stages. Stage I is the initiation portion where damage accumulates and eventually cracking starts and the majority the life of a fatigue test specimen is spent in the initiation stage. In some alloys this stage I portion is distinctive from the rest of the fracture because is consists of faceted fracture. These inclined facets are found to be on slip planes have the highest local critical resolved shear stress. Outside of the faceted area in the stage II portion of the fracture we find the more conventional striated fatigue area on a single plane perpendicular to the applied stress. The presence or absence of facets and other fracture features can be a clue as to what is driving a fatigue crack.

Some alloys make facets easier than others and the faceted areas can range from a single facet to extensive areas of facets. In most wrought alloys single facets can be seen at origins on lower stress low cycle fatigue (LCF) test bars. Since facet formation depends on lower strains the facets are seldom seen at higher stress LCF tests. Among the wrought nickel base superalloys there are a couple alloys worth note that can produce extensive faceted areas. Waspaloy has been known to have large faceted areas at fatigue origins and Incoloy 901 has been known to mix facets and striations in an LCF situation. In a purely high cycle fatigue (HCF) fracture, 901 can exhibit extensive faceted zones but these zones will always be at some extreme angle to the fracture surface and devoid of striations. In conventional alloys, the presence of multiple grains will result in multiple angles of faceting. It should be noted that faceted fracture is not intergranular but a special case of transgranular fracture, where the most active slip planes dictate the plane of fracture. In cast single crystal alloys, facets can sometimes be seen at high cycle fatigue origins and the limitations on their size are not the grain size but other factors. In these single crystal components, a single facet can exist which takes up the entire wall thickness with a neighboring facet at 90 degrees to that facet. These facets in most single crystal alloys tend to operate on the $(1,1,1)$ planes so they are mutually perpendicular.

Since facets can be generated by either failure mode HCF or LCF depending on the alloy and strain level involved, one has to consider the extent, presence, and character of the surrounding stage II propagation when determining if the crack started in HCF or LCF. A wrought part with extensive faceted area which immediately transitions into HCF propagation in the stage II portion most likely cracked in HCF. In the old days of tungsten filament scanning electron microscope (SEM), finding facets and not resolving striations pretty much confirmed HCF. In the absence of the two facet prone alloys, more than one facet is usually a good sign you are heading in the HCF direction. With the field emission guns and in-lens detectors on modern high resolution SEM, fine HCF striations are now readily resolved and one must pay more attention to the rough order of magnitude of the striations to determine if you have HCF or LCF. The number of starts or at least hours of operation of aerospace parts are usually tracked and the maximum number expected for LCF would approximately the number of starts either explicitly tracked 
or estimated from typical cycle times. Striation counts adding up to multiples of this would tend to indicate that that particular crack is driven by high cycle fatigue and this fits with the presence of a large area of facets.

Some of the basic questions about facets include how do facets form and how fast they are generated? Do the dislocations build up until a plane cleaves or can a facet form and then propagate on the same plane in steps? Examining fractures from the field will not help answer these questions completely. Propagation features have been observed and occasional beach marks have been seen that indicate that a facet can be more than a sudden event and that it has a direction of propagation. Another question is do facets always start at the component surface? Experience has shown facets only in clusters that connect to the surface so the surface or very near surface is the prime candidate for initiation location. Experiments involving acoustic emission detection during fatigue testing might be useful as an experimental aid. 